\title{
Etude épidémiologique des nématodes gastro-intestinaux chez les ovins en milieux urbain et périurbain à Maroua, Extrême Nord du Cameroun
}

\author{
R. Ngambia Funkeu ${ }^{1}$ V.S. Pandey ${ }^{1^{*}}$ \\ P. Dorny ${ }^{1}$ S. Killanga ${ }^{2}$
}

Mots-clés

Ovin - Nematoda - Zone urbaine Zone périurbaine - M éthode d'élevage Epidémiologie - Maroua - Cameroun.

\begin{abstract}
Résumé
Une étude épidémiologique a été réalisée sur les nématodes gastro-intestinaux des ovins en milieux urbain et périurbain à Maroua dans la province de l'Extrême Nord du Cameroun où le climat est de type soudano-sahélien avec une saison des pluies et une longue saison sèche. Q uatre systèmes d'élevage ont été identifiés et 111 ovins répartis dans 21 troupeaux ont été suivis sur le plan coprologique et hématologique tous les mois, de novembre 1996 à octobre 1997. Les résultats ont montré la présence des larves infestantes (L3) de cinq genres ou espèces de nématodes : Haemonchus, Cooperia, $\mathrm{O}$ esophagostomum, Trichostrongylus et Strongyloides papillosus. La saison et le type d'élevage ont eu une influence significative $(P<0,05)$ sur l'excrétion d'œufs par gramme de matières fécales (opg). En saison de pluies, les opg ont augmenté dans tous les types d'élevage mais à des degrés plus élevés dans les élevages d'ovins conduits au pâturage en périphérie de la ville et dans ceux d'ovins en claustration permanente. L'hématocrite a été corrélé négativement avec les opg et a diminué significativement en saison de pluies. Des schémas prophylactiques ont été proposés en fonction des systèmes d'élevage et de la saisonnalité du parasitisme.
\end{abstract}

\section{INTRO DUCTION}

Depuis quelques années, un intérêt croissant est accordé aux petits ruminants en Afrique, tant au niveau du développement qu'à celui de la recherche (10). Aussi, en raison de l'expansion démographique urbaine, l'élevage jadis rural gagne progressivement les villes du fait de l'accroissement de la demande citadine en denrées d'origine animale. Ainsi, il a été noté ces dernières années une recrudescence de l'élevage périurbain et urbain (5).

La province de l'Extrême Nord, avec ses 762527 têtes d'ovins, héberge près de la moitié $(46,8$ p. 100) du cheptel ovin recensé au Cameroun en 1989-1990 (19). Dans une enquête menée à Maroua (18), chef-lieu du département du Diamaré et capitale de

1. Institut de médecine tropicale Prince Léopold, Nationalestraat 155, B-2000 Antwerpen, Belgique

2. Institut de la recherche agricole pour le développement (Irad), Centre de Maroua, BP 33, Maroua, Cameroun

* Auteur pour la correspondance

Fax : +32(0)3 2161431 ; e-mail : vpandey@itg.be
l'Extrême Nord, 226 concessions sur 542 visitées étaient détentrices d'animaux. Le rapport ovins/caprins y était de 8,71 contre 0,92 en milieu rural du même département. Ceci témoigne de l'importance qu'occupe l'élevage ovin dans cette ville. Cependant, le développement de la productivité reste limité par les facteurs nutritionnels et pathologiques entre autres (13). Pour la pathologie, les nématodoses demeurent une contrainte non négligeable à cause des pertes de production qu'elles engendrent par des retards de croissance, des troubles de reproduction et des mortalités $(4,13)$. Malgré la présence des services vétérinaires publics et privés, les éleveurs utilisent très rarement les anthelminthiques. Par ailleurs, il n'existe pas d'études longitudinales de longue durée où des informations seraient disponibles sur le parasitisme digestif des ovins en milieux urbain et périurbain, à Maroua ou ailleurs.

Dans les zones urbaines et périurbaines de Maroua, il existe plusieurs modes de conduite de l'élevage qui pourraient influencer le parasitisme. Le présent travail avait pour objectif d'étudier l'évolution saisonnière des nématodes gastro-intestinaux en fonction du mode d'élevage des ovins afin de proposer des schémas prophylactiques qui pourraient contribuer à l'amélioration de la productivité du cheptel. 


\section{MATERIEL ET METHODES}

\section{Zone de l'étude}

Maroua est située entre $10^{\circ} 30^{\prime}$ et $10^{\circ} 40^{\prime}$ de latitude $\mathrm{N}$ et entre $14^{\circ} 20^{\prime}$ et $14^{\circ} 30^{\prime}$ de longitude E. Son climat est de type soudanosahélien de plaine avec une saison des pluies (de juin à octobre) et une saison sèche (de novembre à mai). La couverture végétale est celle d'une savane arborée parsemée d'herbes et de cultures. Les températures maximales $\left(37{ }^{\circ} \mathrm{C}\right)$ sont observées entre mars et mai tandis que les températures minimales $\left(16-18{ }^{\circ} \mathrm{C}\right)$ sont obtenues entre décembre et février. La pluviométrie varie entre 500-1000 mm par an. La ville est traversée longitudinalement par des cours d'eau appelés mayo qui débordent parfois en saison des pluies et tarissent en saison sèche, dès mars.

Durant la période de l'étude, les données pluviométriques et thermiques ont été obtenues auprès du service de météorologie de l'Institut de la recherche agricole pour le développement (Irad, Maroua).

\section{Les animaux}

Cent onze ovins ont été suivis de novembre 1996 à octobre 1997. Ils étaient répartis dans 21 troupeaux localisés dans différentes parties de la ville de Maroua. Ces troupeaux ont été classés en quatre catégories d'élevage en fonction du mode de conduite (tableau I) qui pouvait avoir un effet sur le parasitisme :

- dans le type 1 , les ovins étaient conduits au pâturage, avec des bovins, en périphérie de la ville par un bouvier adulte ;

- dans le type 2, les ovins étaient conduits au pâturage en ville, avec ou sans caprins, par des bergers mineurs ;

- dans le type 3, on laissait divaguer les ovins librement dans le quartier ;

- dans le type 4, les ovins étaient en état de claustration permanente.

Les troupeaux ont été choisis en fonction de leur disponibilité, de leur effectif (cinq moutons au minimum par troupeau) et de leur suivi sanitaire (animaux non déparasités depuis au moins six mois). Cinq à quinze ovins ont été choisis par troupeau en fonction de l'âge.

Les races rencontrées étaient constituées de moutons Peuhls (Foulbé, Poulfouli), Oudah et Massa (Kirdi). La distinction des races n'était pas toujours nette du fait des croisements inter races.
L'alimentation était constituée d'herbes, de feuilles, de fruits d'arbres et de paille sur pied pour les animaux conduits au pâturage, d'herbes, de déchets domestiques et de paille sur pied pour les animaux en divagation, et d'herbes fraîches ou fanées ou de foin pour les animaux en claustration permanente. Parfois les animaux recevaient une complémentation à base de drêches de brasseries locales (bière de sorgho, eau de vie), de tourteaux de coton, de coques de coton, de tige de sorgho, de fanes d'arachide ou de niébé et de fruits d'Acacia.

Les animaux étaient logés la nuit dans de petites cases, des parcs clôturés ou des enclos ouverts, semi-ouverts ou couverts.

\section{Méthodes parasitologiques}

Les matières fécales ont été prélevées au niveau du rectum, une fois par mois, entre 6 et $10 \mathrm{~h}$ du matin. Ces échantillons ont été acheminés au laboratoire dans une glacière. A l'arrivée, ils ont été mis au réfrigérateur et examinés dans les 24 heures qui ont suivi.

L'examen coproscopique a été fait selon la méthode quantitative modifiée de McMaster (7) afin de déterminer le nombre d'œufs de strongles et de Strongyloides par gramme de fèces. Chaque œuf compté correspondait à un opg de 100.

Les coprocultures ont été faites de décembre 1996 à octobre 1997. Les échantillons de fèces ont été mélangés par troupeau, puis mis en culture dans une boîte de pétri en verre pendant huit jours à $31{ }^{\circ} \mathrm{C}$.

L'extraction des larves L3 a été réalisée selon la technique de Baermann (7). L'identification des larves L3 a été faite selon Anon. (11).

\section{Hématocrite}

De janvier à octobre 1997, des échantillons de sang ont été prélevés au niveau de la veine jugulaire, une fois par mois, à l'aide d'un tube vacutainer Venoject contenant de l'héparine-lithium ou du trisodium citrate comme anticoagulant. L'hématocrite a été déterminé au moyen d'une minicentrifugeuse Ames Microspin (M 1101).

\section{Analyses statistiques}

Les données sur l'opg et de l'hématocrite sont exprimées en moyennes géométriques calculées après la transformation logarithmique des données brutes. Les effets de la saison, de l'âge, du sexe, du type d'élevage sur l'opg et l'hématocrite ont été analysés par le modèle linéaire général (glm).

\section{Tableau I}

Distribution des ovins par type d'élevage, ferme, classe d'âge et sexe en milieux urbain et périurbain à Maroua, Extrême Nord du Cameroun

\begin{tabular}{|c|c|c|c|c|c|c|c|}
\hline \multirow[t]{2}{*}{$\begin{array}{c}\text { Type } \\
\text { d'élevage }\end{array}$} & \multirow[t]{2}{*}{$\begin{array}{c}\text { Nombre } \\
\text { d'élevages }\end{array}$} & \multirow[t]{2}{*}{$\begin{array}{l}\text { Nombre } \\
\text { d'ovins }\end{array}$} & \multicolumn{3}{|c|}{ Nombre d'ovins par classe d'âge } & \multicolumn{2}{|c|}{$\begin{array}{c}\text { Nombre } \\
\text { d'ovins par sexe }\end{array}$} \\
\hline & & & $<1$ an & $1-2$ ans & $>2$ ans & mâle & femelle \\
\hline 1 & 3 & 23 & 7 & 5 & 11 & 4 & 19 \\
\hline 2 & 6 & 30 & 14 & 7 & 9 & 6 & 24 \\
\hline 3 & 6 & 29 & 18 & 1 & 10 & 4 & 25 \\
\hline 4 & 6 & 29 & 14 & 5 & 10 & 6 & 23 \\
\hline Total & 21 & 111 & 53 & 18 & 40 & 20 & 91 \\
\hline
\end{tabular}




\section{RESU LTATS}

Les moyennes mensuelles de la pluviométrie et des températures minimales et maximales sont présentées à la figure 1. Des pluies inhabituelles ont été enregistrées aux mois d'avril et de mai. Les précipitations totales de l'année ont été de $701 \mathrm{~mm}$.

Les œufs de nématodes (strongles et Strongyloides) ont été détectés dans les fèces des ovins des quatre types d'élevage. Les moyennes des opg de Strongyloides ont été faibles et ont varié entre 0 et 54, sans fluctuations sensibles. C'est pour cette raison que seules les moyennes des opg de strongles ont été décrites ci-dessous et présentées dans la figure 2 .

La saison et le type d'élevage ont influencé significativement l'opg $(\mathrm{P}<0,05)$. Les valeurs moyennes de l'opg ont augmenté dans tous les élevages en saison de pleines pluies (figure 2). Cette augmentation a été plus marquée dans les élevages d'ovins conduits au pâturage avec les bovins (type 1) et dans les élevages d'ovins en claustration permanente (type 4). Des pics d'opg de $1475,556,666$ et 1444 opg ont été enregistrés en saison des pluies, respectivement dans les élevages $1,2,3$ et 4 .

Les coprocultures ont révélé la présence de cinq genres ou espèces de nématodes parasites : Haemonchus, Trichostrongylus, Cooperia, Oesophagostomum et Strongyloides papillosus. Les larves de Trichostrongylus ont été prépondérantes en saison sèche, celles de Haemonchus en saison de pluies.

Dans tous les types d'élevage, les moyennes de l'hématocrite ont été plus basses en saison de pleines pluies (figure 3). Cette diminution a été plus marquée dans l'élevage de type 1 . L'influence du type d'élevage a été significative $(\mathrm{P}<0,001)$.

L'âge des animaux a influencé significativement $(\mathrm{P}<0,05)$ l'excrétion d'œufs de strongles (tableau II). Les ovins âgés de moins d'un an ont excrété plus d'œufs de novembre à janvier, tandis que les animaux âgés d'un à deux ans ont eu un opg élevé de mai à octobre avec des valeurs moyennes variant de 49 à 694 .

L'âge a influencé significativement l'hématocrite $(\mathrm{P}<0,05)$ (tableau III). Les moyennes mensuelles sont restées relativement stables de janvier à juillet : entre 28 et 31 p. 100 pour les ovins

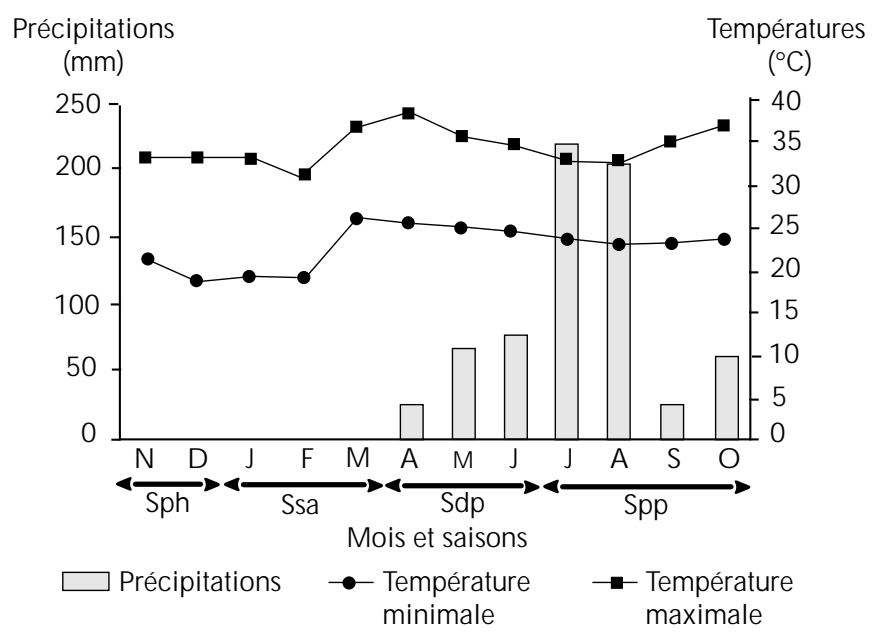

Figure 1 : précipitations et températures moyennes mensuelles à Maroua, Extrême $\mathrm{N}$ ord du Cameroun.

Sph = saison posthivernage $;$ Ssa $=$ saison sèche aride $;$ Sdp $=$ saison de débuts de pluies; $\mathrm{Spp}=$ saison de pleines pluies.
Opg moyen

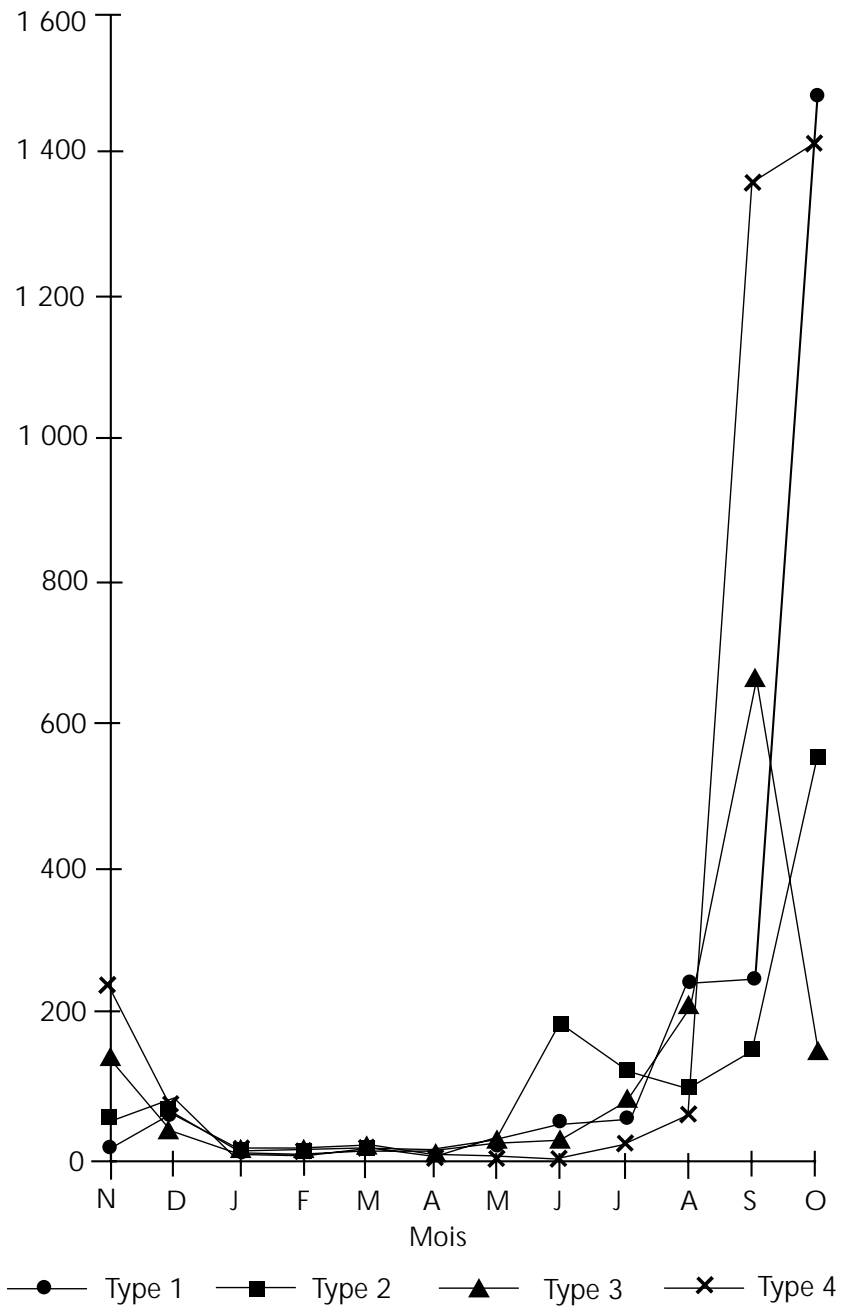

Figure 2 : moyennes géométriques mensuelles des nombres d'œufs de strongles gastro-intestinaux par gramme de fèces (opg) chez les ovins des différents types d'élevage urbains et périurbains à Maroua, Extrême $\mathrm{N}$ ord du Cameroun.

Type 1 = ovins conduits au pâturage avec des bovins en périphérie de la ville ; type 2 = ovins conduits au pâturage en ville ; type $3=$ ovins en divagation libre dans le quartier; type 4 = ovins en claustration permanente.

âgés jusqu'à 2 ans et entre 27 et 29 p. 100 pour les animaux âgés de plus de 2 ans. D'août à octobre, les valeurs ont diminué et se sont situées entre 20 et 24 p. 100 chez les ovins de moins d'un an, entre 21 et 23 p. 100 chez les ovins âgés d'un à 2 ans et entre 19 et 22 p. 100 chez les ovins âgés de plus de 2 ans. Le sexe n'a pas eu d'effet significatif sur l'hématocrite.

\section{DISCUSSIO N}

Cette étude a montré la présence d'infestations aux nématodes gastro-intestinaux des ovins en milieux urbain et périurbain. La saisonnalité de l'excrétion d'œufs de strongles dans les fèces des ovins a été marquée dans tous les systèmes d'élevage. En effet, les moyennes géométriques des opg mensuels ont été faibles en saison sèche (saison posthivernage, saison sèche aride) et au début des pluies. Lors des pleines pluies, elles ont connu des hausses notoires dont les plus importantes ont été observées dans les éle- 


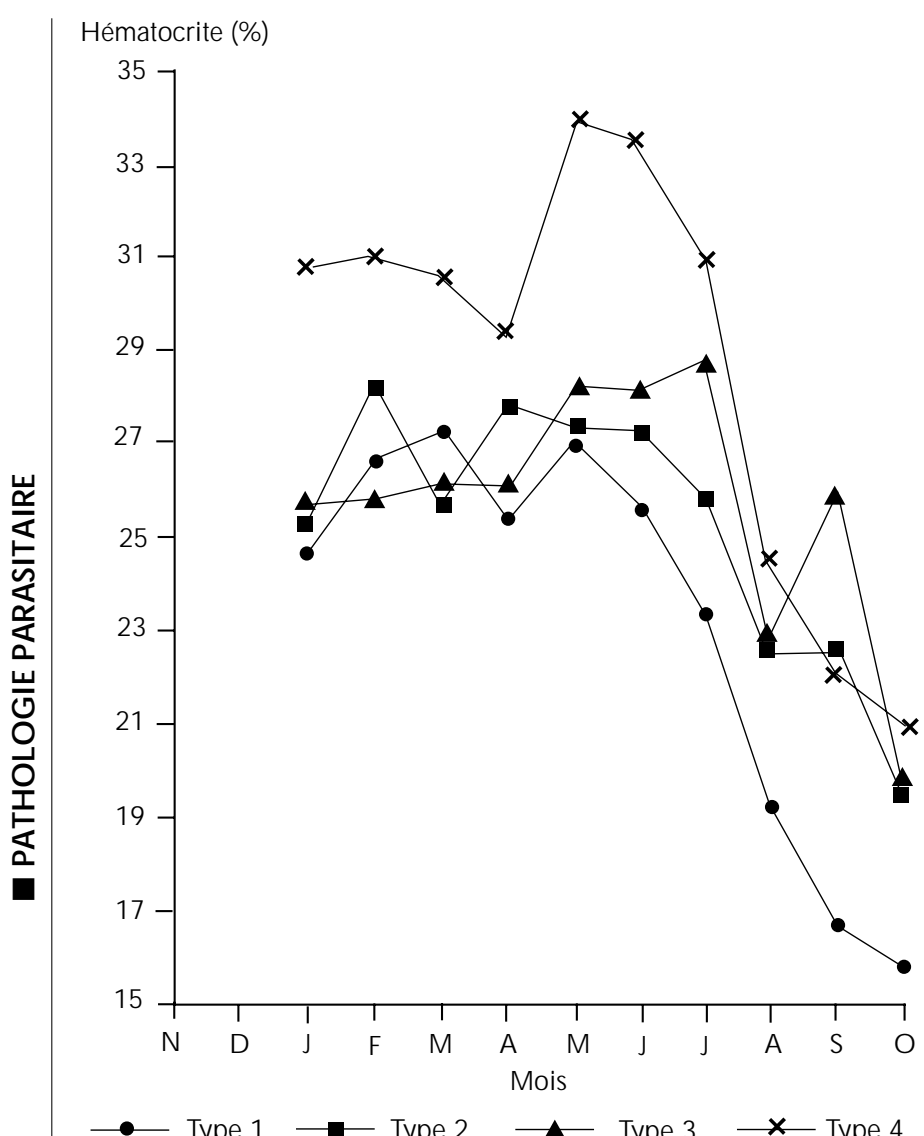

Figure 3 : moyennes géométriques mensuelles de l'hématocrite chez les ovins des différents types d'élevage urbains et périurbains à M aroua, Extrême N ord du Cameroun.

Type 1 = ovins conduits au pâturage avec des bovins en périphérie de la ville; type 2 = ovins conduits au pâturage en ville ; type 3 = ovins en divagation libre dans le quartier; type 4 $=$ ovins en claustration permanente. vages de types 1 et 4 . Cette évolution saisonnière a été observée sous des climats similaires en Afrique de l'Ouest, notamment en Gambie (1), au Nigeria (16), au Sénégal (12) et au Togo (3). En saison sèche, les conditions sont défavorables au développement et à la survie des formes libres de parasites (6). Par conséquent, il y a diminution des risques d'infection des animaux, comme cela a été constaté par Ankers et coll. (1). L'humidité est un facteur important dans le développement et la survie des nématodes gastro-intestinaux des ruminants $(2,15)$.

Une différence significative de l'intensité des infections entre différents systèmes d'élevage a été enregistrée. L'augmentation de l'opg en saison des pluies a été plus marquée dans les élevages d'ovins conduits au pâturage avec les bovins (type 1) et dans les élevages d'ovins en claustration permanente (type 4). Les ovins des élevages de type 1 auraient été plus exposés à l'infestation car, outre l'infection probable sur le pâturage, ils auraient pu être également infectés lors de leur abreuvement dans des points d'eau de pluie ou tout au long des cours d'eau où la densité des animaux, en transit dans la ville ou séjournant là quelque temps avant leur abattage, est plus grande. Les ovins en claustration permanente (élevage de type 4) ont été très probablement contaminés par le biais des herbes fraîches distribuées à l'auge, ces herbes provenant généralement d'aires ou paissaient d'autres ruminants. Aussi, l'apport de ces herbes dans l'alimentation des animaux, qui se fait à un stade avancé de la saison des pluies, pourrait expliquer l'augmentation tardive (à partir de juillet) de l'opg. L'augmentation plus modérée des opg chez les ovins conduits aux herbes en ville (élevage de type 2 ) et chez les ovins en divagation libre (élevage de type 3) serait due à la restriction de leurs sorties en saison des pluies, période des cultures et des récoltes. Une chute de l'hématocrite a coïncidé avec l'augmentation des opg en saison des pluies. Elle peut être expliquée par l'action hématophage de $H$. contortus qui était le nématode prépondérant dès que les premières pluies tombaient. La chute de l'hématocrite a été plus marquée dans les élevages de type 1 ; il a été noté qu'en saison de pleines pluies les tiques ont été très abondantes sur les animaux de ces élevages et auraient ainsi eu un effet sur l'hématocrite.

\section{Tableau II}

Moyennes géométriques mensuelles et valeurs extrêmes des opg de strongles en fonction de l'âge des ovins en milieux urbain et périurbain à Maroua, Extrême $\mathrm{N}$ ord du Cameroun

\begin{tabular}{lrlcccc} 
& \multicolumn{2}{c}{$<$} & \multicolumn{2}{c}{$\mathbf{1 - 2}$ ans } & \multicolumn{2}{c}{ an ans } \\
\cline { 2 - 5 } & Moyenne & Extrêmes & Moyenne & Extrêmes & Moyenne Extrêmes \\
Novembre & 143 & $0-1500$ & 46 & $0-400$ & 51 & $0-3700$ \\
Décembre & 97 & $0-2500$ & 23 & $0-500$ & 58 & $0-4200$ \\
Janvier & 7 & $0-500$ & 3 & $0-400$ & 5 & $0-900$ \\
Février & 4 & $0-300$ & 5 & $0-500$ & 6 & $0-700$ \\
Mars & 16 & $0-1400$ & 16 & $0-900$ & 8 & $0-1700$ \\
Avril & 5 & $0-300$ & 10 & $0-400$ & 3 & $0-2800$ \\
Mai & 3 & $0-1600$ & 37 & $0-3200$ & 64 & $0-2300$ \\
Juin & 18 & $0-3400$ & 49 & $0-2400$ & 51 & $0-4600$ \\
Juillet & 37 & $0-2400$ & 223 & $0-5200$ & 63 & $0-6500$ \\
Août & 101 & $0-12100$ & 419 & $0-3500$ & 120 & $0-7200$ \\
Septembre & 492 & $0-5500$ & 554 & $0-12200$ & 238 & $0-7000$ \\
Octobre & 539 & $0-3100$ & 694 & $0-1000$ & 680 & $0-3700$
\end{tabular}


Tableau III

M oyennes géométriques mensuelles et valeurs extrêmes de l'hématocrite en fonction de l'âge des ovins à Maroua, Extrême Nord du Cameroun

\begin{tabular}{|c|c|c|c|c|c|c|}
\hline & \multicolumn{2}{|c|}{$<1$ an } & \multicolumn{2}{|c|}{ 1-2 ans } & \multicolumn{2}{|c|}{$>2$ ans } \\
\hline & Moyenne & Extrêmes & Moyenne & Extrêmes & Moyenne & Extrêmes \\
\hline Janvier & 28 & $16-46$ & 28 & $16-37$ & 27 & $17-41$ \\
\hline Février & 29 & $16-44$ & 29 & $24-40$ & 29 & $20-46$ \\
\hline Mars & 29 & $20-36$ & 28 & $12-44$ & 28 & $22-46$ \\
\hline Avril & 28 & $22-34$ & 30 & $24-36$ & 28 & $20-40$ \\
\hline Mai & 31 & $22-48$ & 31 & $22-48$ & 29 & $18-42$ \\
\hline Juin & 30 & $22-48$ & 33 & $24-48$ & 27 & $20-46$ \\
\hline Juillet & 29 & $16-42$ & 28 & $18-38$ & 28 & $16-42$ \\
\hline Août & 24 & $18-32$ & 23 & $16-36$ & 22 & $14-30$ \\
\hline Septembre & 24 & $12-38$ & 23 & $12-36$ & 22 & $12-28$ \\
\hline Octobre & 20 & $18-28$ & 21 & $12-28$ & 19 & $12-28$ \\
\hline
\end{tabular}

\section{CONCLUSION}

Dans les différents systèmes d'élevage en milieux urbain et périurbain, les nématodes sévissent, notamment $H$. contortus, Cooperia spp., O. columbianum, S. papillosus et Trichostrongylus spp. Les opg sont faibles à modérées en saison sèche selon les descriptions de Hansen et Perry (8) et augmentent en saison de pluies. Cette augmentation est plus marquée chez les ovins conduits au pâturage périurbain avec les bovins et chez les ovins en claustration permanente.

Sur le plan épidémiologique, une vermifugation collective en fin de saison des pluies dans les élevages de types 1 et 4 est souhaitable pour réduire les charges parasitaires. Dans les élevages de types 2 et 3 , les auteurs suggèrent un traitement individuel lors de cas cliniques. A l'entrée de la saison des pluies, un traitement peut être effectué chez les animaux jeunes et chez les brebis en fin de gestation. Les anthelminthiques utilisés doivent être efficaces aussi bien contre les stades adultes que les larves de parasites. Il reste à faire une étude de l'opportunité économique de cette prophylaxie.

\section{BIBLIO GRAPHIE}

1. ANKERS P., ZINSSTAG J., PFISTER K., 1994. Quasi-absence de réinfestation par les strongles du bétail gambien en saison sèche. Revue Elev. M éd. vét. Pays trop., $47:$ 201-205.

2. BERBIGIER P., GRUNER L., MAMBRINI M., SOPHIE S.A., 1990. Faecal water content and egg survival of gastrointestinal strongles under dry tropical conditions in Guadeloupe. Parasitol. Res., 76: 379-385.

3. BONFOH B., ZINSSTAG J., ANKERS P., PANGUI L.J., PFISTER K., 1995. Epidémiologie des nématodes gastro-intestinaux chez les petits ruminants dans la région des plateaux au Togo. Revue Elev. Méd. vét. Pays trop., 48 : 321-326.

4. FABIYI J.P., 1987. Production losses and control of helminths in ruminants of tropical regions. Int. J. Parasitol., 17: 435-442.

5. FAYE B., PLANCHENAULT D., 1996. Socio-économie de l'élevage périurbain : Secoville. Rapport scientifique à mi-parcours, Lomé, 6-16 mai 1996. Montpellier, France, Cirad-emvt, $152 \mathrm{p}$.

6. FRITSCHE T., KAUFMANN J., PFISTER K., 1993. Parasite spectrum and seasonal epidemiology of gastrointestinal nematodes of small ruminants in The Gambia. Vet. Parasitol., 49: 271-283.
7. GRABER M., PERROTIN G., 1983. Helminthes et helminthoses des ruminants domestiques d'Afrique tropicale. Maisons-Alfort, France, le Point vétérinaire, $378 \mathrm{p}$.

8. HANSEN J., PERRY B., 1974. The epidemiology, diagnosis and control of helminth parasites of ruminants. A handbook. Rome, Italy, FAO/ILRAD, $171 \mathrm{p}$.

9. KOMOIN-OKA C., ZIN SSTAG J., PANDEY V.S., FO FANA F., N'DEPO A., 1999. Epidémiologie des parasites des ovins de la zone sud forestière de la Côte d'Ivoire. Revue Elev. M éd. vét. Pays trop., 52 : 39-46.

10. LANDAIS E., 1985. Problèmes liés au développement de l'élevage des petits ruminants (ovins et caprins) en Afrique. Rapport de synthèses. In: $6^{\mathrm{e}}$ conférence de la commission régionale de I'OIE pour l'Afrique, Harare, Zimbabwe, 22-25 janvier 1985. Paris, France, OIE, p. 19-170.

11. Manual of veterinary parasitological laboratory techniques. Reference book 418, 3rd Ed., 1986. London, UK, Ministry of Agriculture, Fisheries and Food, Her Majesty's Stationary O ffice, $160 \mathrm{p}$.

12. NDAO M., BELOT J., ZINSSTAG K., PFISTER K., 1992 Epidémiologie des nématodes gastro-intestinaux des ruminants dans la zone sylvopastorale au Sénégal. In : $7^{\mathrm{e}}$ Conférence internationale des institutions de médecine vétérinaire tropicale, Aimvt, Yamoussoukro, Côte-d'Ivoire, 2 : 485-489.

13. NJOYA A., AWA N.D., BOUCHEL D., 1997. Influence de la complémentation et de la prophylaxie sur la viabilité des ovins Foulbé au Nord-Cameroun. Revue Elev. M éd. vét. Pays trop., 50 : 227-233.

14. OUATTARA L., OUEDRAOGO A., KAUFMANN J., PFISTER K., 1992. Epidémiologie des nématodes gastro-intestinaux des ruminants aux Burkina Faso. In : $7^{\mathrm{e}}$ Conférence internationale des institutions de médecine vétérinaire tropicale, Aimvt, Yamoussoukro, Côte-d'Ivoire, 2 497-505.

15. ROSSANIGO C.E., GRUNER L., 1994. Relative effect of temperature and moisture on the development of strongyle eggs to infective larvae in bovine pats in Argentina. Vet. Rec., 55: 317-325.

16. SCHILLHORN VAN VEEN T.W., 1978. Haemonchosis in sheep during the dry season in the Nigerian savana. Vet. Rec., 102: 364-365.

17. STATA CORP, 1999. Stata statistical software: Release 6.0. College Station, TX, USA, Stata Corporation.

18. THYS E., EKEMBE T., 1992. Elevage citadin des petits ruminants à Maroua (Province de l'Extrême N ord Cameroun). Cah. Agric., 1 : 249-255.

19. YEM YEM Z., 1992. Principaux facteurs limitants de la santé animale au Cameroun. In $7^{\mathrm{e}}$ Conférence internationale des institutions de médecine vétérinaire tropicale, Aimvt, Yamoussoukro, Côte-d'Ivoire, 2 561-573.

Reçu le 06.08.99, accepté le 03.10 .00 


\section{Summary}

N gambia Funkeu R., Pandey V.S., Dorny P., Killanga S Epidemiological study of gastrointestinal nematodes of sheep in urban and suburban areas, in Marua, Extreme North of Cameroon

An epidemiological study was carried out on gastrointestinal nematodes of sheep in urban and suburban areas, in Marua, located in the Extreme North province of Cameroon, where the climate is of the Sudano-Sahelian type with a short rainy season and a long dry season. Four animal husbandry systems were identified. From November 1996 to O ctober 1997, 111 sheep belonging to 21 flocks were monitored monthly for coprological and hematological evaluations. Results showed the presence of infective larvae (L3) of five genera/species of nematodes: Haemonchus, Cooperia, O esophagostomum, Trichostrongylus and Strongyloides papillosus. The season and animal husbandry system had a significant influence on the eggs per gram of feces count (EPG) $(P<0.05)$. EPGs increased during the rainy season in all animal husbandry systems, but were higher in flocks that grazed on pastures at the periphery of the town and in those kept permanently confined. The packed cell volume showed a negative correlation with EPG and significantly decreased during the rainy season. Prophylactic schemes are suggested in relation with the animal husbandry system and seasonal variation of parasite activity.

Key words: Sheep - N ematoda - U rban area - Suburban area Animal husbandry method - Epidemiology - Marua Cameroon.

\section{Resumen}

N gambia Funkeu R., Pandey V.S., D orny P., Killanga S. Estudio epidemiológico de los nemátodos gastrointestinales en los ovinos en medio urbano y peri urbano en Marua, Extremo Norte de Camerún

Se realizó un estudio epidemiológico sobre los nemátodos gastrointestinales de los ovinos en medio urbano y peri urbano en Marua en la provincia del Extremo Norte de Camerún, donde el clima es de tipo sudano-sahariano, con una estación de lluvias y una larga estación seca. Se identificaron cuatro sistemas de crianza y se siguieron 111 ovinos repartidos en 21 hatos, tanto desde el punto de vista coprológico como hematológico todos los meses, de noviembre 1996 a octubre 1997. Los resultados muestran la presencia de larvae infestantes (L3) de cinco géneros o especies de nemátodos: Haemonchus, Cooperia, O esophagostomum, Trichostrongylus y Strongyloides papillosus. La estación y el tipo de crianza tuvieron influencia significativa $(P<0,05)$ sobre la excreción de huevos por gramo de materia fecal (OPG). Durante la estación de lluvias, la OPG aumentó en todos los tipos de crianza, pero en niveles más elevados en los criaderos de ovinos conducidos a pastizales en la periferia de la ciudad y en aquellos ovinos en encierro permanente. El hematocrito estuvo correlacionado negativamente con la OPG y disminuyó significativamente durante la estación de lluvias. Se proponen esquemas profilácticos en función de los sistemas de crianza y de la estacionalidad de los parásitos.

Palabras clave : O vino - Nematoda - Zona urbana - Zona periurbana - Epidemiología - Marua - Camerún. 